\title{
Interaction of the Flames of Diluted Methane-Oxygen Mixtures with Obstacles of Conical Shape
}

\author{
N.M. Rubtsov ${ }^{1}$, G.I. Tsvetkov ${ }^{1}$, V.I. Chernysh ${ }^{1}$,K.Ya. Troshin ${ }^{5}$, \\ ${ }^{1}$ Institute of Structural Makrokinetics and Materials Science, Russian Academy of Sciences, \\ Moscow region, Chernogolovka, 142432, Russia \\ ${ }^{2}$ N.N. Semenov Institute of Chemical Physics, Russian Academy of Sciences, \\ Moscow, 119991, Russia \\ nmrubtss@mail.ru
}

\begin{abstract}
It is experimentally shown that the flame of methane-oxygen mix does not get through the central opening of a confuser up to the opening angle of $83^{\circ}$, but gets through the central opening of the diffuser, despite of the existence of additional openings on cone elements. The results of numerical modeling with the use of the equations of Navier-Stokes in the compressible reacting environment in acoustic approach allowed to describe qualitative features of flame propagation through an obstacle of conical shape with additional openings on cone elements.
\end{abstract}

Keywords: flame, methane-oxygen mixture, obstacle, speed filming, combustion, obstacles of conical shape, numerical modeling

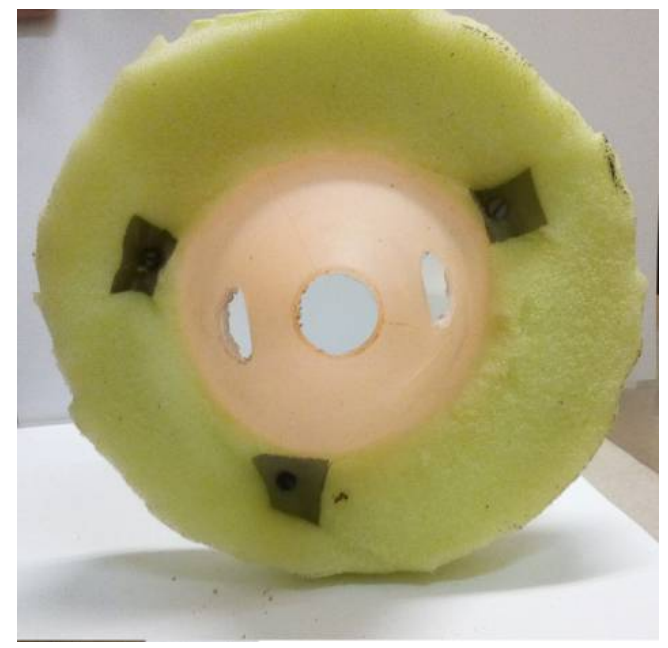

The obstacle of conical shape with three openings (opening angle $83^{\circ}$ ) 


\title{
Взаимодействие пламён разбавленных метано - кислородных смесей с препятствиями конической формы
}

\author{
Н.М. Рубцов ${ }^{1}$, Г.И. Цветков ${ }^{1}$, В.И.Черныш ${ }^{1}$, К.Я. Трошин \\ ${ }^{1}$ Институт Структурной Макрокинетики и Проблем Материаловедения РАН, \\ Россия, Московская область, г. Черноголовка, 142432, Институтская ул., 8 \\ ${ }^{2}$ Институт химической физики им. Н.Н.Семенова РАН, \\ Россия, Москва, 119991, ул. Косьгина, 4 \\ nmrubtss@mail.ru
}

\begin{abstract}
Аннотация
Экспериментально показано, что пламя разбавленной метано-кислородной смеси не проникает через центральное отверстие конфузора до угла раскрытия $83^{0}$. Такое пламя проникает через центральное отверстие диффузора, даже в случае наличия дополнительных отверстий на образующих конуса. Результаты численного моделирования с использованием уравнений Навье-Стокса в сжимаемой реагирующей среде в акустическом приближении позволили описать качественные особенности распространения пламени через препятствие конической формы с дополнительными отверстиями на образующих конуса.
\end{abstract}

Ключевые слова: скоростная киносьемка, горение, пламя, препятствия конической формы, метано-кислородная смесь, численное моделирование

\section{1. Введение}

Взрывы метана, сопровождающиеся распространением пламени, до сих пор представляют угрозу помещениям химических заводов, шахтным тоннелям, трубам и других объёмах в устройствах хранения и транспортировки горючих газов. Нештатные ситуации, связанные с воспламенениями в перерабатывающих отраслях промышленности могут привести к потерям человеческих жизней и повреждениям производственных помещений [1-4]. Одна из проблем взрывобезопасности состоит в том, чтобы устранить или минимизировать последствия случайных воспламенений и взрывов в трубах. Для достижения этой цели необходимо иметь достоверные экспериментальные данные при моделировании взрывов метана в трубах [5], в производственных помещениях, камерах сгорания и химических реакторах, которые представляют собой объемы сложной геометрии. Моделирование турбулентного дефлаграционного горения заранее перемешанных горючих смесей в замкнутом объёме сложной геометрии является непростой проблемой, особенно в связи с необходимостью адекватного представления зоны реакции, что предполагает некоторый эксперимент. В связи с этим моделирование в малых объёмах при сравнительно низких давлениях является весьма желательным для прогнозирования ожидаемых в большом масштабе эффектов и значительно менее затратно [6]. Кроме того, такое моделирование позволяет определить, представляет ли натурный эксперимент в большем масштабе при атмосферном давлении опасность для целостности установки и для жизни персонала.

При взрывах газа, имеющих место в перерабатывающих отраслях промышленности, число и местоположение отверстий в препятствиях являются параметрами (наряду с другими, например, формой препятствия, величиной относительного отверстия и т.д.), которые 
определяют интенсивность таких взрывов [7, 8]. В литературе существует весьма ограниченная информация о роли этих параметров [9].

При моделировании процессов дозвукового турбулентного горения приближение малого числа Маха (акустическое приближение) для уравнений Навье-Стокса в сжимаемой реагирующей среде является удовлетворительныой основой для моделирования на качественном уровне $[9,10]$. Тепловыделение при горении создает нестабильность потоков газа при его расширении, что в свою очередь ускоряет переход от ламинарного к турбулентному горению. Турбулентность также увеличивает скорость горения за счёт ускорения смешивания. В нашем рассмотрении пламя заранее перемешанной смеси распространяется сначала как ламинарный фронт, который возмущается при проникновении через препятствия и становится турбулентным пламенем, распространяющимся с более высокой скоростью.

Мы ранее показали, что пламя разбавленной метано-кислородной смеси не проходит через конфузор с единственным отверстием в центре, при этом пламя свободно проникает через диффузор. Это означает, что при тех же самых условиях предел проникновения метанокислородного пламени через диффузор много меньше, чем в случае конфузора, поэтому конфузор представляет собой наиболее эффективное физическое устройство для подавления пламени [11]. Данные по особенностям проникновения пламени через коническое препятствие с центральным отверстием при наличии дополнительных отверстий на образующих конуса, в литературе отсутствуют. Такие отверстия могут позволить сбросить давление, возникающее при приближении фронта пламенени к стенке и продлить время эксплуатации конфузора как устройства, подавляющего пламя.

Мы ранее показали, что максимальное давление и максимальная акустическая интенсивность намного больше при проникновении пламени через плоское препятствие с несколькими отверстиями по сравнению с плоским препятствиием с единственным отверстием [12], т.е. два отверстия и тем более три являются более эффективными турбулизаторами, чем единственное отверстие..

В данной работе установлены особенности распространения пламени через конус с центральным отверстием и отверстиями на образующих, ориентированный как конфузор и как диффузор. Осуществлено качественное численное моделирование наблюдаемых закономерностей на основе уравнений Навье-Стокса в сжимаемой реагирующей среде в приближении малого числа Маха. Таким образом, в работе рассмотрена модель, учитывающая относительный вклад газодинамических и химических факторов в горении, которая может быть использована для моделирования распространения пламени через препятствия различной геометрии.

\section{2. Эксперимент}

Эксперименты проводили со стехиометрическими смесями метана с кислородом, разбавленными углекислым газом $\mathrm{CO}_{2}$ и аргоном $\mathrm{Ar}$ при начальных давлениях $150 \div 200$ Торр и начальной температуре $298 \mathrm{~K}$ в горизонтально расположенном цилиндрическом кварцевом реакторе 70 см длиной и 14 см в диаметре. Два электрода искрового воспламенения (энергия разряда 3 Дж) были расположены в торце реактора. Реактор был установлен в двух шлюзах из нержавеющей стали, снабжённых отверстиями для напуска и откачки газа и дверцей безопасности, которая открывалась наружу, если общее давление в реакторе превышало 1 атм $[9,11,12]$. Пластиковая воронка $d=14$ см с центральным отверстием и двумя отверстиями (каждое - 17 мм в диаметре) на образующих конуса (углы раскрытия конусов составляли 450, 550 и 830 (рис. 1)) была ориентирована как диффузор или как конфузор и помещена в центре реактора. Препятствие фиксировали в реакторе кольцом пенополиуретана (рис. 1). Горючую смесь $\left(15.4 \% \mathrm{CH}_{4}+30.8 \% \mathrm{O}_{2}+46 \% \mathrm{CO}_{2}+7.8 \% \mathrm{Ar}\right)$ составляли до эксперимента; $\mathrm{CO}_{2}$ добавляли, чтобы уменьшить скорость фронта пламени и улучшить качество съемки; $\mathrm{Ar}$ добавляли, чтобы уменьшить порог инициирования искрового разряда. 


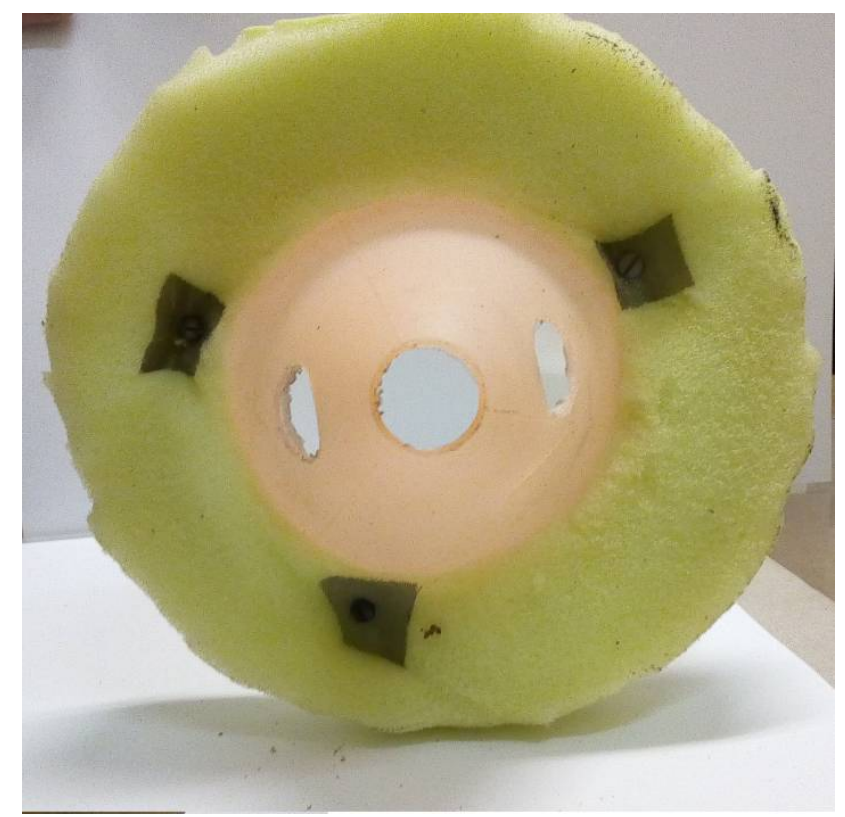

Рис.1. Препятствие конической формы с тремя отверстиями (угол раскрытия $83^{0}$ )

Реактор заполняли смесью до необходимого давления. Затем осуществляли искровой разряд. Скоростную киносъемку динамики воспламенения и распространения фронта пламени осуществляли с боковой стороны реактора $[9,11,12]$ с помощью цветной цифровой камеры Casio Exilim F1 Pro (частота кадров $600 \mathrm{c}^{-1}$ ). Одновременную регистрацию радикалов $\mathrm{CH}\left(\mathrm{A}^{1} \Delta-\mathrm{X}^{2} \Pi\right)$ при $431 \mathrm{~nm}[13]$ осуществляли с использованием второй скоростной кинока-

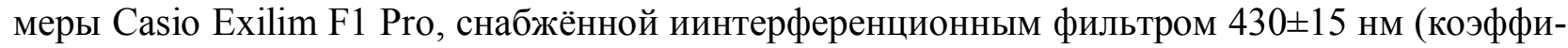
циент пропускания 40\% при 430 нм). Видеофайлы сохраняли в памяти компьютера и затем проводили их покадровую обработку. Изменение давления в ходе сгорания регистрировали пьезоэлектрическим датчиком, сиигнал которого сиинхронизировали с искровым разрядом. Акустические колебания регистрировали с использованием чувствительного микрофона Ritmix (чувствительность до 40 кГц). Аудиофайл сохраняли в памяти компьютера и анализировали с помощью пакета программ Spectra Plus 5.0. Газы использовали марки хч.

Типичные последовательности видеокадров скоростной съемки распространения фронта пламени в горючей смеси при начальном давлении 165 Торр через коническое препятствие, ориентированное как конфузор а), в), г) и как диффузор б), д), е) представлены на рис. 2 для углов раскрытия конуса $55^{0}$ и $83^{0}$. Как видно из рис. 2 , в наших условиях пламя всегда проникает только через центральное отверстие диффузора. При этом в случае конфузора пламя проникает только через его боковые отверстия при угле раскрытия конуса $55^{0}$ (рис. 2, а, кадры 21, 22, рис. 2, в, кадр 19). Проникновение пламени через конфузор сопровождается с резким и громким звуком, при этом дверца безопасности открывается. Укажем, что распространение пламени через диффузор не сопровождается резким звуковым эффектом, дверца безопасности не открывается. На рис. 3 представлены иллюстрирующие сказанное выше зависимости акустической амплитуды от времени при проникновении пламени через препятствие в виде конфузора (рис. 3, а) и диффузора (рис. 3, б). Несколько видеокадров из рис. 2а и рис. 26 приведены на рис. 3; центр каждого видеокадра соответствует текущему времени. Из полученных выше результатов можно заключить, что натурный эксперимент с конфузором с углом раскрытия менее $83^{0}$ в большом масштабе при атмосферном давлении опасен для целостности установки и для жизни персонала, т.е. его проведение нецелесообразно. 


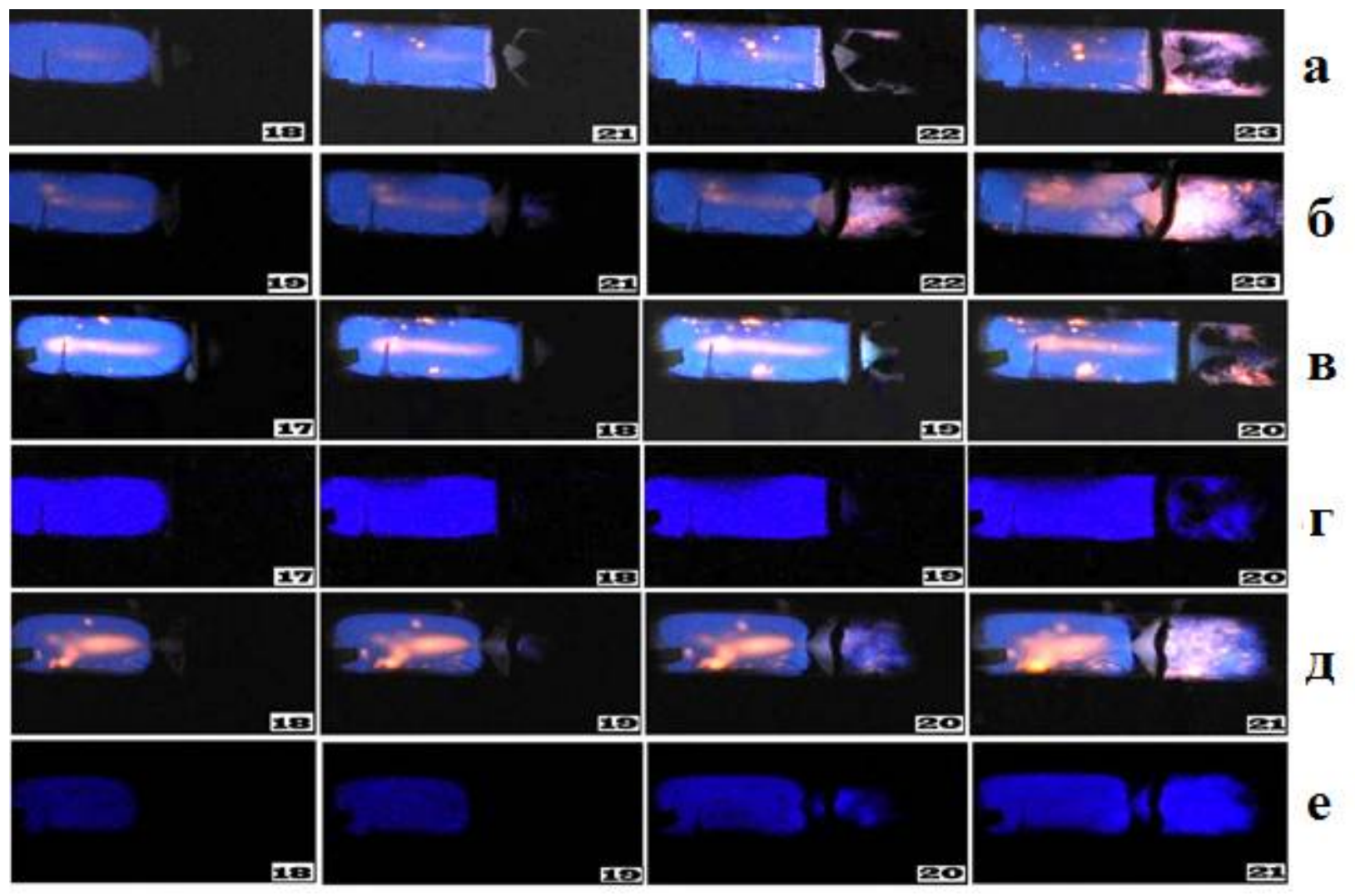

Рис. 2. Видеокадры скоростной киносъёмки распространения фронта пламени через коническое препятствие с центральным отверстием и двумя отверстиями на образующих конуса:

а) конфузор (угол раскрытия конуса $55^{0}$ );

б) диффузор (угол раскрытия конуса $55^{\circ}$ );

в) конфузор (угол раскрытия конуса $83^{0}$ ),

г) конфузор (угол раскрытия конуса $83^{0}$ ), интерференционный фильтр 430 нм помещен перед видеокамерой;

д) диффузор (угол раскрытия конуса $83^{0}$ );

е) диффузор (угол раскрытия конуса $83^{\circ}$ ), интерференционный фильтр 430 нм помещен перед видеокамерой.

Начальное давление 165 Торр. Число на кадре соответствует порядковому номеру видеокадра после момента инициирования

С увеличением угла раскрытия пламя начинает проникать через центральное отверстие диффузора (рис. 2, в, кадры 19, 20). Отметим, что диаметры отверстий в коническом препятствии значительно меньше, чем минимальный диаметр проникновения пламени через плоское препятствие с единственным центральным отверстием (20 мм [11]). Поэтому при оценке пожарной безопасности помещения с несколькими проёмами величину минимального размера единственного отверстия использовать не следует, поскольку при увеличении количества отверстий диаметр отверстия, достаточного для проникновения пламени, уменьшается.

Отметим также, что в случае плоского препятствия с тремя отверстиями (угол раскрытия, очевидно, $180^{\circ}$ ) пламя проникает через каждое из этих трех отверстий [12]. В нашем случае в конической полости возникают отраженные акустические волны, что сопровождается появлением застойных зон и взаимодействием этих волн с исходным фронтом горения, который сгенерировал эти волны; максимальное давление при этом регистрируется на некотором расстоянии от вершины конуса [13].

Укажем также, что с уменьшением угла раскрытия в конфузоре с центральным отверстием (угол раскрытия менее $45^{0}$ ) пламя не проникает и через центральное отверстие независимо от существования дополнительных отверстий на образующих конуса. 


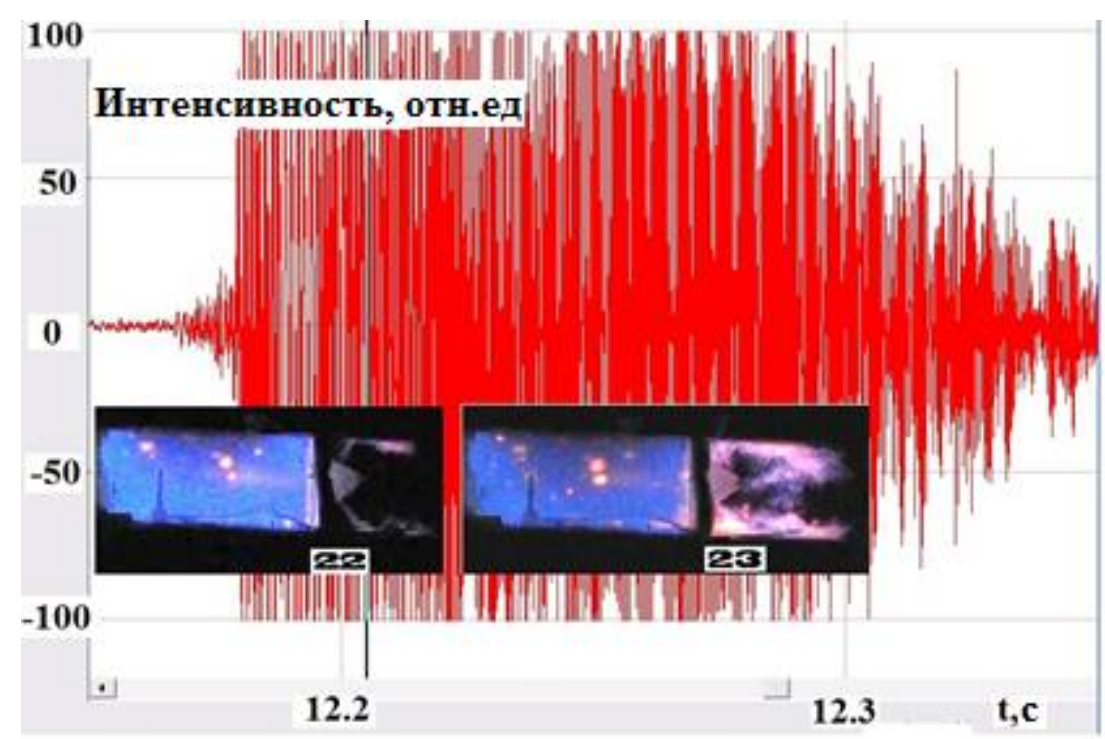

$\mathrm{a}$

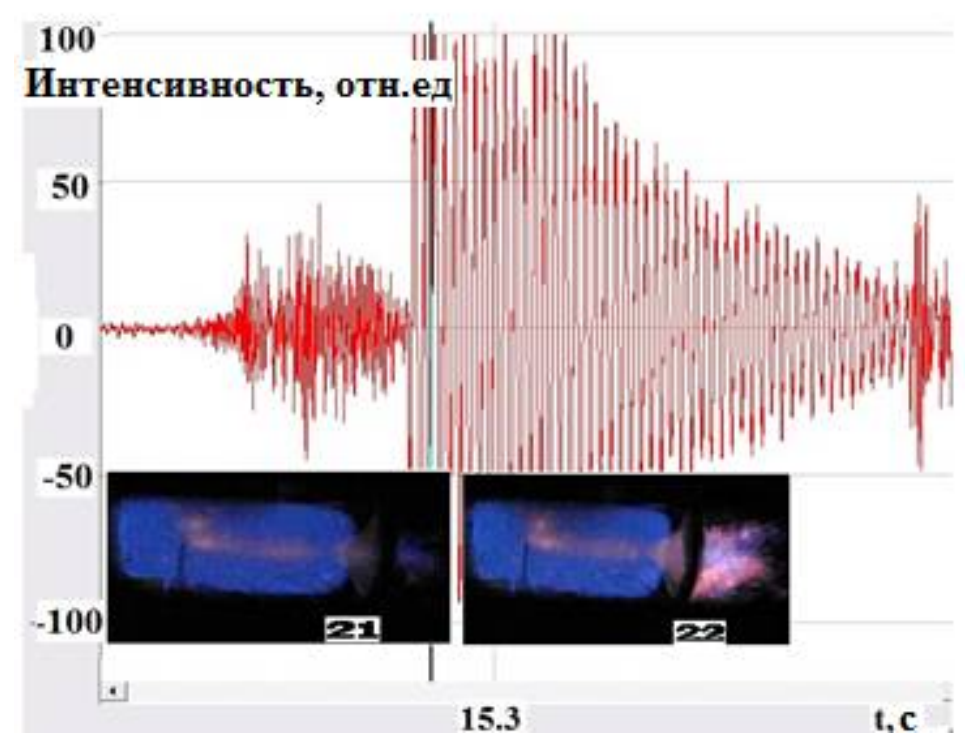

Рис. 3. Зависимости амплитуд акустических колебаний от времени при распространении пламени в газовой смеси при начальном давлении 165 Торр.

а) конфузор, приведено несколько кадров из рис. 2 , а, центр каждого кадра соответствует текущему времени;

б) диффузор, приведено несколько кадров из рис. 2, б, центр каждого кадра соответствует текущему времени

\section{3. Результаты и обсуждение}

В настоящей работе при описании распространения пламени в двумерном канале осуществлено численное моделирование с использованием уравнений Навье-Стокса для сжимаемой реагирующей среды в приближении малого числа Маха $[14,15]$ показавшее качественное согласие с экспериментами [9, 11, 12]; система уравнений представлена в [11]. Решение проблемы было выполнено методом конечных элементов с помощью пакета (FlexPDE 6.08, 1996-2008 PDE Solutions Inc.[16]). Условие инициирования было взято как $T=10$ на границе канала; в середине канала находилось препятствие конической формы с двумя дополнительными отверстиями на образующих конуса. Граничными условиями (включая препятствие) были $C_{x}=0, C_{y}=0, n=0, u=0, v=0, \rho_{x}=0, \rho_{y}=0$, а также условие 
конвективного теплообмена $T_{t}=T-T_{0}$ (индексы $x, y, t$ означают дифференцирование по $x, y$, $t$ ). Химическая реакция была представлена единственной реакцией Аррениуса первого порядка [11]. Имеется в виду уравнение (ж) системы (1.II), стр. 9 работы [11]. Там же на стр.8 указано, каким образом химическая реакция представляется одностадийной реакцией Аррениуса первого порядка в безразмерных переменных.

Качественные результаты численного моделирования процесса проникновения пламени через коническое препятствие в виде конфузора и диффузора приведены на рис. 4.

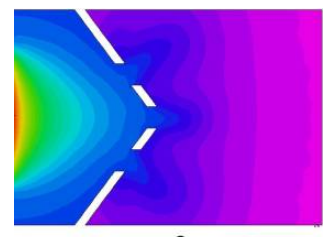

$7.10^{-3}$

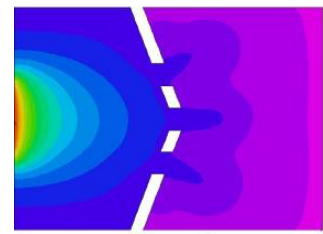

$8.71 .10^{-3}$

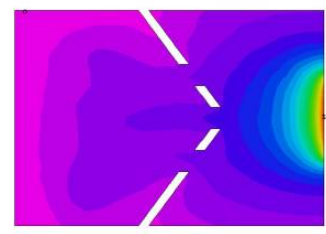

0.0151

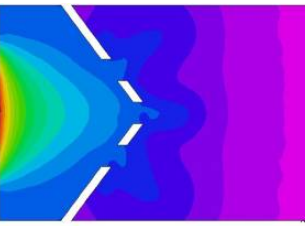

$8.19 .10^{-3}$

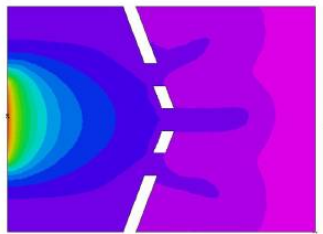

9.43.10 ${ }^{-3}$

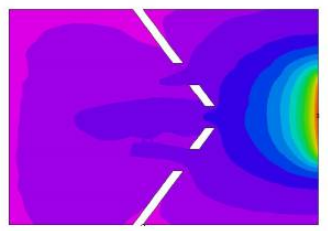

0.0160

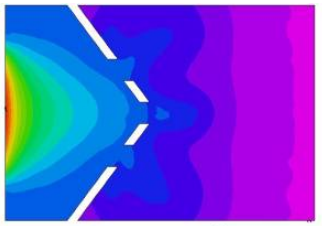

$8.21 .10^{-3}$

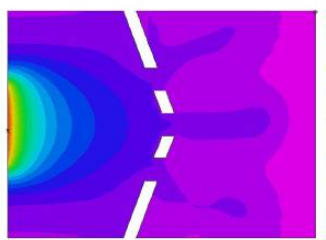

$9.75 .10^{-3}$

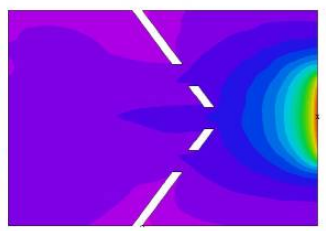

0.0165

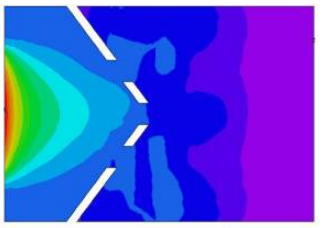

8.56.10 ${ }^{-3}$ t, c
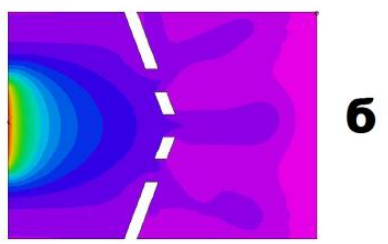

18

12

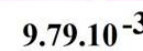

t, c

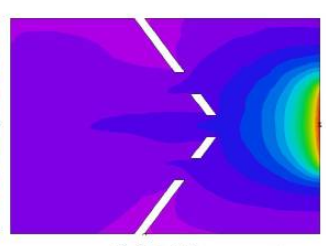

0.0168

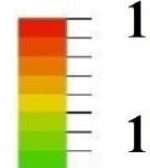

a

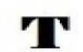

6

B

1

Рис. 4. Результаты численного расчёта процесса проникновения пламени через препятствие конической формы.

a) изменение безразмерной температуры при проникновении пламени через конфузор, угол раскрытия $100^{\circ}$;

б) изменение безразмерной температуры при распространении пламени через конфузор, угол раскрытия $150^{\circ}$;

в) изменение безразмерной температуры при распространении пламени через диффузор, вводный угол раскрытия $100^{\circ}$.

Шкала безразмерной температуры представлена справа

Как видно, результаты вычислений находятся в качественном согласии с экспериментами, приведёнными на рис. 2, а именно, пламя проникает через диффузор (рис. 4, в); пламя не проникает через центральное отверстие конфузора с углом раскрытия $100^{0}$ (рис. 4, a). При большем угле раскрытия $\left(150^{0}\right.$, рис. 4,6$)$ пламя проникает через все три отверстия в конфузоре в качественном согласии с экспериментом (см. рис. 2). Отметим, что в случае плоского препятствия с тремя отверстиями (один из них - центральное, угол раскрытия $-180^{\circ}$ ), пламя проникает через каждое из этих трех отверстий [12]. Очевидно, что качественное рассмотрение (например, единственная реакция вместо полного химического механизма, двухмерное моделирование и т.д.) не позволяет получить точное значение угла раскрытия, при котором пламя начинает проникать через центральное отверстие конфузора. Кроме того, такое качественное отличие от процесса проникновения пламени через плоское препятствие с центральным отверстием указывает на значимую роль взаимодействия акустических колебаний реактора, содержащего коническую полость, с распространяющимся фронтом горения [17] даже в случае дозвукового пламени. 


\section{4. Заключение}

В рамках приближённого рассмотрения с использованием уравнений Навье-Стокса в сжимаемой реагирующей среде качественно описаны особенности распространения пламени через препятствие конической формы с дополнительными отверстиями на образующих конфузора, а именно то, что пламя не проникает через центральное отверстие конфузора, но проникает только через центральное отверстие диффузора, даже при наличии отверстий на образующих конуса. Проведённое моделирование в малых объёмах позволяет предположить, что в случае нештатной ситуации через открытый вентиль, расположенный в центре конфузора, находящегося в трубе, пламя не проникнет. При этом наиболее эффективным двухсторонним пламяпреградителем в трубе может служить система из двух конфузоров, воронки которых расположены на оси трубы по потоку газа и против него, поскольку аварийная ситуация может произойти до и после препятствия. Посредине могут располагаться отверстие или вентиль.

В части изучения горения при использовании скоростной цветной киносъемки работа выполнена в рамках государственного заказа № гос. рег. АААА-А17-117011910011-09, в части изучения горения смесей водород-метан-воздух - в рамках государственного заказа AAAA-A17-117040610346-5.

\section{Литература}

1. Kundu S., Zanganeh, J., Moghtaderi B. J. A review on understanding explosions from methane-air mixture.//Loss Prev. Process Ind. 2016. Vol. 40, pp. 507-523.

2. Zhang B., Pang L.,Gao Y. Detonation limits in binary fuel blends of methane/hydrogen mixtures, // Fuel. 2016. Vol. 168. pp. 27-33.

3. Lowesmith B.J., Hankinson G., Johnson D.M. Vapour cloud explosions in a long congested region involving methane/hydrogen mixtures // Process Safety and Environmental Protection. 2011. Vol. 89. pp. 234-247.

4. Pekalski A., Puttock J., Chynoweth S. J. Deflagration to detonation transition in a vapour cloud explosion in open but congested space // Journal of Loss Prevention in the Process Industries 2015. Vol. 36. Pp. 365-370.

5. Lees' Loss Prevention in the Process Industries: Hazard Identification, Assessment and Control. Butterworth-Heinemann. 2012. 3680 p.

6. Виноградов А.Н., Калинин А.П., Родионов И.Д., Родионов А.И, Родионова И.П., Рубцов Н.М., Черныш В.И., Цветков Г.И., Трошин К.Я. Устройство для дистанционного изучения процессов горения и взрыва с использованием гиперспектрометрии и скоростной фотосъемки. Полезная модель. Патент №158856 от 22.07.2015 Опубликовано 20.01.2016 Бюл. № 2.

7. Gubba S.R., Ibrahim S.S., Maladasekera W., Masri A. R. LES modeling of premixed deflagrating flames in a small-scale vented explosion chamber with a series of solid obstructions // Combustion Science and Technology 2008. Vol. 180. Pp. 1936-1955.

8. Kindracki J., Kobiera A., Rarata G., Wolanski P. Influence of ignition position and obstacles on explosion development in methane-air mixture in closed vessels // Journal of Loss Prevention in the Process Industries. 2007. Vol. 20. Pp. 551-561.

9. Nickolai.M.Rubtsov, The Modes of Gaseous Combustion, Springer International Publishing. 2016. 297 p.

10. Akkerman V., Bychkov V., Petchenko A., Eriksson L.-E., Flame oscillations in tubes with nonslip at the walls // Combustion and Flame. 2006. Vol. 145. Pp. 675-678. 
11. Рубцов Н.М., Виноградов А.Н., Калинин А.П., Родионов А.И., Родионов И.Д., Трошин К.Я., Цветков Г.И., Черныш В.И. Установление газодинамических и кинетических особенностей проникновения метано-кислородных пламён через препятствия путем использования 4D спектроскопии и скоростной киносъёмки//Физико-химическая кинетика в газовой динамике. 2016. T.17, вып. 4. http://chemphys.edu.ru/issues/2016-17-4/articles/661/

12. Рубцов. Н.М., Калинин А.П., Цветков Г.И., Трошин К.Я. Особенности взаимодействия фронта горения с плоскими препятствиями на примере пламён разбавленных метано-кислородных смесей//Физико-химическая кинетика в газовой динамике. 2018. Т. 19, вып. 1. http://chemphys.edu.ru/issues/2018-19-1/articles/723/

13. Lewis B., Von Elbe G. Combustion, Explosions and Flame in Gases. New York, London. Acad. Press. 1987. $731 \mathrm{p}$.

14. Majda A. Equations for Low Mach Number Combustion. Center of Pure and Applied Mathematics. University of California. Berkeley. 1982, PAM-112.

15. Nicoud F. Conservative High-Order Finite-Difference Schemes for Low-Mach Number Flows // Journal of Computational Physics. 2000. Vol. 158. Pp. 71-97.

16. Backstrom G. Simple Fields of Physics by Finite Element Analysis. GB Publishing, 2005. 324 p.

17. О. А. Васильев, Фокусировка сильной ударной волны при отражении от вогнутых поверхностей// Матем. моделирование, 1996, Т.8, С.115-120.

Статья поступила в редакцию 10 декабря 2018 г. 